\title{
Interferometric measurements of piezooptic coefficients by means of four-point bending method
}

\author{
Krupych O., Savaryn V., Skab I. and Vlokh R. \\ Institute of Physical Optics, 23 Dragomanov St., 79005 Lviv, Ukraine \\ E-mail: ok@ifo.lviv.ua
}

Received: 14.07 .2011

\begin{abstract}
A new technique is suggested for determination of piezooptic coefficients, which represents a combination of digital imaging laser interferometry and a canonical four-point bending method. The design of interferometer, measurement procedures, and data processing are described in detail. Potentials of the present technique are tested on the example of widely used optical glass BK7. High enough precision, together with unambiguity in determination of the sign of both piezooptic $\left(\pi_{q m}\right)$ and photoelastic $\left(p_{q m}\right)$ coefficients, allow us to claim it to be one of the most accurate and reliable techniques for the measurements of $\pi_{q m}$ and $p_{q m}$ parameters.
\end{abstract}

Keywords: piezooptic effect, interferometry, measuring techniques

PACS: $78.20 . \mathrm{Hp}, 42.25 . \mathrm{Hz}$

UDC: $535.551,535.417$

\section{Introduction}

Photoelastic effect consists in changes of refractive indices of optical media in the presence of mechanical stresses. First studied by David Brewster in the early 19th century [1], it is widely applied for determination of mechanical stresses (photoelasticity), in optical remote stress sensors, accelerometers, stress tensor field tomography, light polarisation modulators, etc. (see, e.g., [2-7]). One of the most important applications of the photoelasic effect is concerned with widely explored acoustooptic devices [8-11]. Thereby the knowledge of accurate values of piezooptic parameters for different practical materials is of great importance.

Historically, the first description of the photoelastic effect given by D. Brewster (see Ref. [1]) was given for isotropic solids and cubic crystals. According to this formulation, an optical birefringence $\Delta n$ induced by a mechanical stress $\sigma$ is expressed as:

$$
\Delta n=K \sigma \text {. }
$$

A coefficient $K$ is termed as a Brewster's constant, relative piezooptic coefficient, or a stress optical coefficient. The latter term comprises a standard specification used by manufacturers of optical glasses [12]. As a matter of fact, Eq. (1) is applicable only for isotropic optical media. For a more general case of anisotropic materials, a tensor relation suggested by F. Pockels [13] has to be used:

$$
\delta B_{i j}=\sum_{k=1}^{3} \sum_{l=1}^{3} \pi_{i j k l} \sigma_{k l} \quad(i, j, k, l=1,2,3),
$$

where $\delta B_{i j}$ are the increments of optical impermeability tensor components $B_{i j}$ (a constitutive second-rank tensor), $\sigma_{k l}$ the components of stress tensor (a polar second-rank tensor), and $\pi_{i j k l}$ the components of fourth-rank tensor of piezooptic coefficients. Internal symmetry of the tensors involved here ( $B_{i j}=B_{j i}$ and $\sigma_{k l}=\sigma_{l k}$ ) allows one to write out Eq. (2) in the matrix form:

$150 \quad$ Ukr. J. Phys. Opt. 2011, V12, №3




$$
\delta B_{q}=\pi_{q m} \sigma_{m}(q, m=1 \ldots 6),
$$

where $\sigma_{1}=\sigma_{11}, \sigma_{2}=\sigma_{22}, \sigma_{3}=\sigma_{33}, \sigma_{4}=\sigma_{23}, \sigma_{5}=\sigma_{13}$, and $\sigma_{6}=\sigma_{12}$, while $\delta B_{1}=\delta B_{11} \delta B_{2}=\delta B_{22}$, $\delta B_{3}=\delta B_{33}, \delta B_{4}=\delta B_{23}, \delta B_{5}=\delta B_{13}$, and $\delta B_{6}=\delta B_{12}$. We have $\pi_{q m}=\pi_{i j k l}$ if $m=1,2,3$ and $\pi_{q m}=2 \pi_{i j k l}$ if $m=4,5,6$. Notice that the Einstein summation convention is used hereafter.

Eqs. (2) and (3) are mathematical formulations of the piezooptic effect. When the increment of optical impermeability tensor is expressed in terms of mechanical strains, a term "elastooptic effect" or "strain-optical effect" is conventionally used. In the matrix form, this effect may be written as follows:

$$
\delta B_{q}=p_{q m} \varepsilon_{m}(q, m=1 \ldots 6),
$$

where $p_{q m}$ and $\varepsilon_{m}$ are the components of fourth-rank tensor of elastoooptic coefficients and secondrank strain tensor, respectively. Here the strain and stress tensors are related via the Hook's law, i.e.:

$$
\varepsilon_{q}=S_{q m} \sigma_{m}, \quad \sigma_{q}=C_{q m} \varepsilon_{m}
$$

where $S_{q m}$ coefficients represent the fourth-rank compliance tensor, and $C_{q m}$ the fourth-rank stiffness tensor (or the elasticity tensor). Thus, the piezooptic and elastooptic coefficients are related through the compliance or stiffness tensors:

$$
\pi_{q m}=p_{q l} S_{l m}, \quad p_{q m}=\pi_{q l} C_{l m} .
$$

Unfortunately, the piezooptic coefficients $\pi_{q m}$ are usually measured with significant errors, which often exceed tens of per cent (among many works demonstrating this fact, see, e.g., [1416]). As shown in Ref. [17], the main cause of high measurement errors for the piezooptic coefficients is due to inhomogeneous 3D distribution of stress components taking place, instead of a homogeneous stress state usually assumed by an idealised model for uniaxially pressed parallelepiped-shape samples. In its turn, the stress inhomogeneity originates from friction forces appearing at contacting sample surfaces, and misalignments in mechanical loading. This friction force hinders free transverse deformation of sample faces, thus leading to barrel-shaped distortion of this sample and inevitable appearance of all the stress tensor components, in spite of the fact that a uniaxial pressure has been initially applied. Besides, the actual mean stress magnitude along light ray travelling through a sample centre differs considerably (by $\sim 10 \%$ ) from its value estimated in the assumption of homogeneous stress state. As a result, the values obtained experimentally for the piezooptic coefficients differ from their true values. It is evident that a real 3D stress distribution in samples depends strongly on the loading conditions and, moreover, the worst still is that these conditions differ from one experiment to another or from laboratory to laboratory. This means that application of laborious theoretical calculations of exact 3D stress fields, combined with rigorously uniaxial sample loading, seem to represent unrealistic way when improving determination of the piezooptic coefficients.

A possible alternative to the approach described above is suggested by the loading methods that induce inhomogeneous though a priori known stress distributions in samples. A well-known four-point bending of a bar and diametrically-stressed disk methods [18] are among them. Another promising modern technique is associated with torsion of crystal rods [19, 20]. In this work we describe a four-point bending method combined with digital imaging laser interferometry for determination of the piezooptic coefficients.

Ukr. J. Phys. Opt. 2011, V12, №3 


\section{Four-point bending method}

A canonical four-point bending method induces pure bending [21]. A schematic draw that clarifies application of force in frame of this method is shown in Fig. 1. The stress components in the central part of sample (within the upper loading span) are expressed as follows:

$$
\sigma_{1} \equiv \sigma_{x}=\frac{6 P a}{b h^{3}} y, \quad \sigma_{2} \equiv \sigma_{y}=0, \quad \sigma_{3} \equiv \sigma_{z}=0,
$$

where $P$ is the loading force, $h$ and $b$ the sample height and depth, respectively, $a$ the distance between the planes where the forces act. It follows from Eq. (1) that only one stress tensor component, $\sigma_{1}$, is nonzero in the central part of sample. Its magnitude varies from $\sigma_{1}=-\frac{3 P a}{b h^{2}}$ at the upper face $(y=-h / 2)$, through $\sigma_{1}=0$ at the neutral line $(y=0)$, to $\sigma_{1}=+\frac{3 P a}{b h^{2}}$ on the bottom

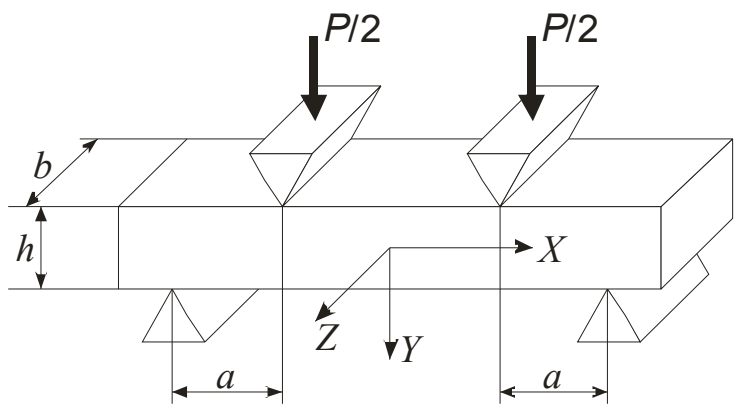

Fig. 1. Schematic view of sample-loading geometry according to four-point bending technique. A Cartesian coordinate system is depicted, too. $P$ is loading force. Light beam propagates along $Z$ direction.

face $(y=h / 2)$. Such geometry implies that the upper sample face is compressed whereas the bottom one expanded, with equal strength modules. It is worth noticing that, according to Eq. (7), the stress component $\sigma_{1}$ depends linearly upon the coordinate $y$. The slope of this dependence is determined by geometrical parameters and the loading force.

\section{Interferometer setup and measuring procedures}

In this work we use a Mach-Zehnder type interferometer equipped with a CCD camera for recording an interference pattern (see Fig. 2). A He-Ne laser (1) emits a polarised light (the wavelength of $\lambda=632.8 \mathrm{~nm}$ ), which passes through a circular polariser (2). Rotations of this polariser allow smooth attenuation of light intensity. A beam expander (4) provides a wide collimated beam. A polariser (5) is used to switch between the horizontal and vertical polarisations and a prism (6) splits the beam into two channels. A probing beam propagates through a sample (7). Being reflected by mirrors (8), both the probing and reference beams are superposed by a second prism (6). An objective lens (9) builds a sample image on a CCD camera matrix. As a result, sharp sample images with clear interference patterns can be obtained. The CCD image is digitised and stored with a computer (11). Finally, switching of light polarisation is performed by a motorised rotary stage governed by a controller (12).

The optical phase difference between the arms of interferometer is as follows:

$$
\Delta \Phi=\frac{2 \pi}{\lambda} b\left(n-n_{m}\right)
$$

where $b$ is the sample thickness along $Z$ axis, and $n$ and $n_{m}$ are the refractive indices of sample and 


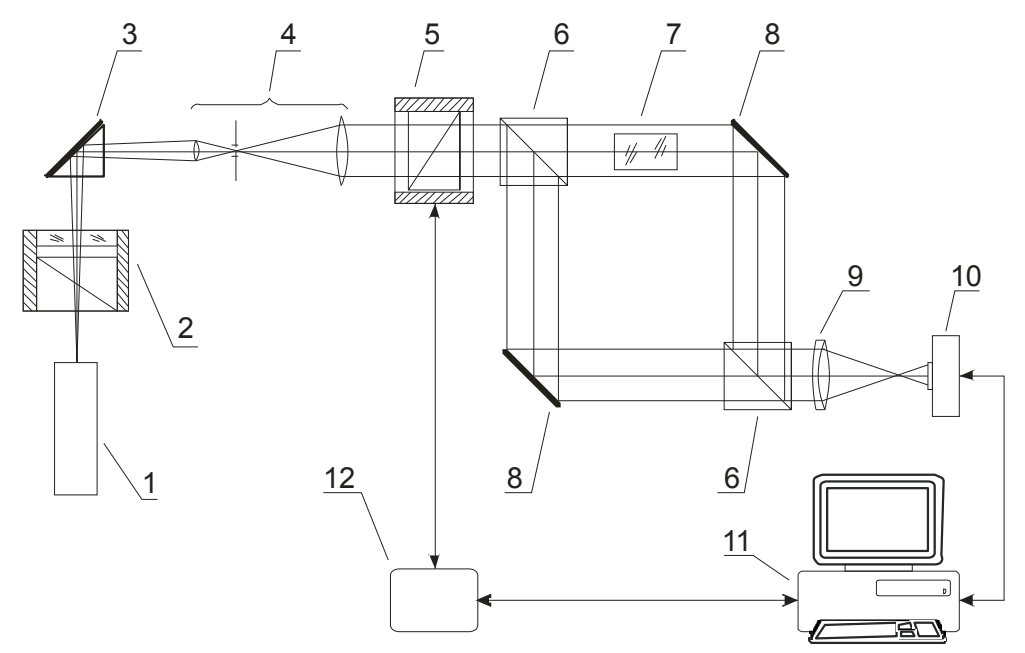

Fig. 2. Interferometer setup: (1) He-Ne laser; (2) circular polariser; (3) right angle prism; (4) beam expander; (5) linear polariser (Glan prism) with motorised rotary stage; (6) beam-splitting prism; (7) sample under test; (8) mirror; (9) objective lens; (10) CCD camera; (11) computer; (12) stepper motor controller.

surrounding medium, respectively. Since $n_{m}=1$ for the air, the phase difference increment $\delta \Phi$ induced by the loading reads as

$$
\begin{aligned}
\delta \Phi & =\Delta \Phi_{1}-\Delta \Phi_{0}=\frac{2 \pi}{\lambda}\left[b^{\prime}\left(n^{\prime}-1\right)-b(n-1)\right]=\frac{2 \pi}{\lambda}[(b+\delta b)(n+\delta n-1)-b(n-1)]= \\
& =\frac{2 \pi}{\lambda}[\delta n(b+\delta b)+\delta b(n-1)],
\end{aligned}
$$

where $\delta n$ is the refractive index increment due to the piezooptic effect, and $\delta b$ the Poisson's elongation (or contraction) along the $Z$ direction. Then the refractive index increments are given by

$$
\delta n_{1}=K_{\|} \sigma_{1}=\left(-\frac{1}{2} n_{0}^{3} \pi_{11}\right) \sigma_{1}, \quad \delta n_{2}=K_{\perp} \sigma_{1}=\left(-\frac{1}{2} n_{0}^{3} \pi_{12}\right) \sigma_{1} .
$$

where $K_{\|}$and $K_{\perp}$ are the photoelastic coefficients (sometimes denoted as $C_{1}$ and $C_{2}$ ), and $\pi_{11}$ and $\pi_{12}$ the piezooptic coefficients. It is worthwhile to notice that the stress optical coefficient $K$ in Eq. (1) is defined by the difference between the photoelastic coefficients $K_{\|}$and $K_{\perp}\left(K=K_{\|}-K_{\perp}\right)$. As seen from Eqs. (10), the coefficients $\pi_{11}$ and $K_{\|}$could be derived when dealing with the horizontal input polarisation, and coefficients $\pi_{12}$ and $K_{\perp}$ for the vertical polarisation. A change in the sample thickness can be expressed as

$$
\delta b=\varepsilon_{3} b=\frac{1}{E}\left[\sigma_{z}-v\left(\sigma_{1}+\sigma_{2}\right)\right] b=-\frac{v}{E} \sigma_{1} b,
$$

where $\varepsilon_{3}$ is the strain tensor component, $v$ the Poisson's ratio, and $E$ the Young's modulus. Let us now estimate the magnitude of $\delta b$. We have used the sample with the dimensions $b=5.65 \mathrm{~mm}$, $h=3.22 \mathrm{~mm}$, and length $=20 \mathrm{~mm}$, made of an optical glass BK7 (according to Schott's classification). The mechanical parameters of the latter are $v=0.206$ and $E=82 \mathrm{GPa}$ [22], and the loading parameters are given by $a=4 \mathrm{~mm}$ and $P=80.9 \mathrm{~N}$. Thus, the maximal stress values at the top and bottom facets $(y= \pm h / 2)$ calculated with Eq. (7) are estimated as $\left|\sigma_{1}^{\max }\right|=\frac{3 P a}{b h^{2}} \approx 17 \mathrm{MPa}=1.7 \times 10^{7} \mathrm{~Pa}$. As a result, we obtain 


$$
\delta b \leq 4.2 \times 10^{-5} \mathrm{~mm}, \quad \frac{\delta b}{b}<10^{-5} .
$$

Due to the fact that $\delta b \ll b$, Eq. (9) could be reduced to the form

$$
\delta \Phi=\frac{2 \pi}{\lambda}[\delta n \times b+\delta b(n-1)],
$$

which is similar to the optical path change quoted in Ref. [23]. Combining Eqs. (10), (11) and (13), we get

$$
\begin{aligned}
& \delta \Phi_{1}=\frac{2 \pi}{\lambda} b\left[\frac{1}{2} n^{3} \pi_{11}-\frac{v}{E}(n-1)\right] \sigma_{1}=\frac{2 \pi}{\lambda} b C_{1}^{\text {eff }} \sigma_{1}, \\
& \delta \Phi_{2}=\frac{2 \pi}{\lambda} b\left[\frac{1}{2} n^{3} \pi_{12}-\frac{v}{E}(n-1)\right] \sigma_{1}=\frac{2 \pi}{\lambda} b C_{2}^{\text {eff }} \sigma_{1},
\end{aligned}
$$

where $C_{i}^{e f f}=\left(K_{i}+D\right)=\left[-\frac{1}{2} n^{3} \pi_{1 i}-\frac{v}{E}(n-1)\right]$ is the effective photoelastic coefficient, $K_{i}=-\frac{1}{2} n^{3} \pi_{1 i}$ the photoelastic coefficient, and $D=-\frac{v}{E}(n-1)$ the strain-related term. Considering Eqs. (7) and (14), we arrive at the following dependences of the phase difference increments $\delta \Phi$ on the coordinate $y$ :

$$
\delta \Phi_{1}(y)=\frac{12 \pi P a}{\lambda h^{3}} C_{1}^{e f f} y, \quad \delta \Phi_{2}(y)=\frac{12 \pi P a}{\lambda h^{3}} C_{2}^{\text {eff }} y .
$$

Eqs. (15) could be used for experimental determination of the effective photoelastic coefficients.

The measuring procedures include the next steps. First, the input polarisation is switched to the vertical position and an interferogram for the free sample is recorded. Then the loading force is applied and a relevant interferogram is recorded again. The same procedures are repeated for the horizontal input polarisation. Typical interferograms of the central part of sample are presented in Fig. 3.
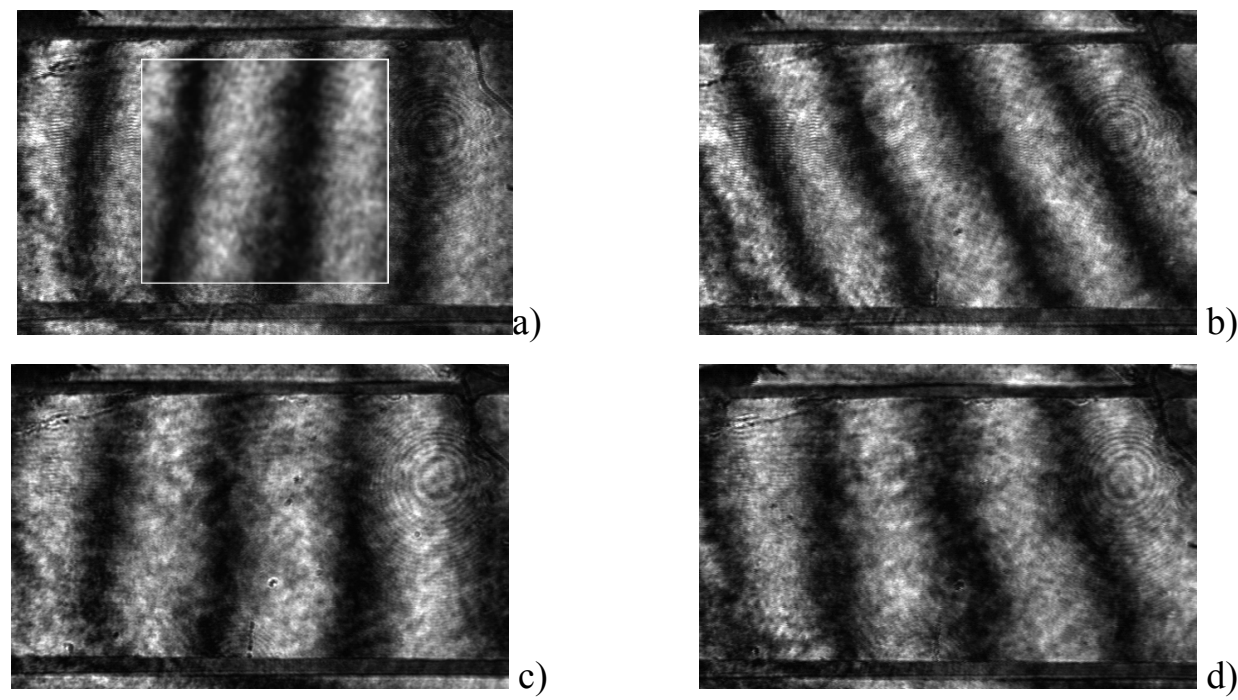

Fig. 3. Typical interferograms obtained in our studies under conditions of (a) free sample, vertical polarisation; (b) loaded sample, vertical polarisation; (c) free sample, horizontal polarisation; (d) loaded sample, horizontal polarisation. Loading force is equal to $80.9 \mathrm{~N}$, field of view $3.65 \times 5.58 \mathrm{~mm}^{2}$, and image size $361 \times 600$ pixels. The area selected in figure (a) corresponds to a region under analysis and contains smoothed data. 


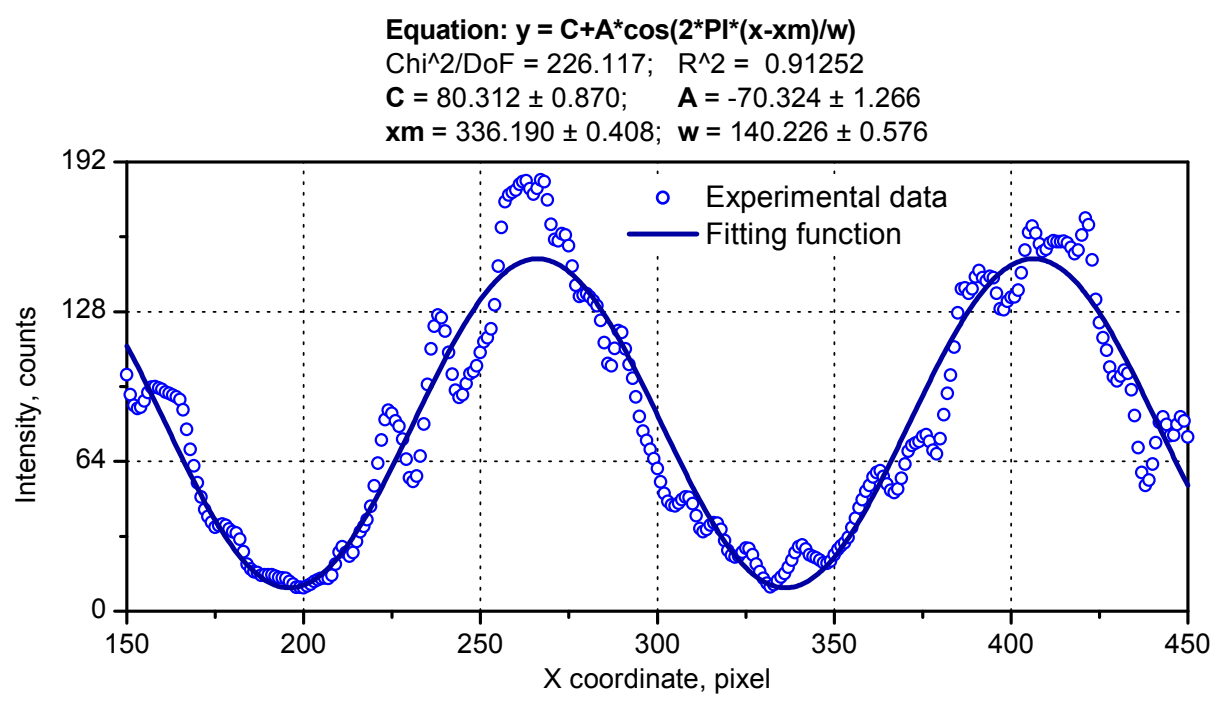

Fig. 4. An example of experimental data fitted by sinusoidal function. Fitting results and statistics are presented in the legend.

Processing of interferograms starts with selecting a region inside the sample image, which will be used for calculating the appropriate data. Then a smoothing filter $(3 \times 3$ or $5 \times 5$ pixels $)$ is applied to reduce noises and the intensity data $I$ for the first row of the selected region is fitted by a sinusoidal function, with some amplitude $A$, period $w$, and extremum position $x_{m}$ :

$$
I=C+A \cos \left[\frac{2 \pi}{w}\left(x-x_{m}\right)\right],
$$

with $C$ being the mean intensity level. An example of data fitting for a one row is depicted in Fig. 4. The parameters $w$ and $x_{m}$ obtained from the fitting procedure are used when calculating the optical phase difference $\Delta \Phi_{2}$ (here the index "2" means vertical polarisation of the input light). This value is calculated for a certain $x_{c}$ coordinate:

$$
\Delta \Phi_{2}=\frac{2 \pi}{w}\left(x_{c}-x_{m}\right) \text {. }
$$

In this work we have chosen the $x_{c}$ coordinate to be located exactly in the centre of the selected region $\left(x_{c}=300\right.$ pixels). The procedure is repeated for each $5^{\text {th }}$ row $\left(0^{\text {th }}, 5^{\text {th }}, 10^{\text {th }}, \ldots\right)$ of the selected region and the dependence of $\Delta \Phi$ on the $y$ coordinate is obtained. Calculating the $\Delta \Phi_{2}(y)$ dependences for both free $\left(\Delta \Phi_{20}(y)\right)$ and loaded $\left(\Delta \Phi_{21}(y)\right)$ samples, one gets the coordinate dependence of the phase difference increment, $\delta \Phi_{2}(y)=\Delta \Phi_{21}(y)-\Delta \Phi_{20}(y)$.

In the next stage, the input light polarisation is switched to horizontal and the experimental procedure is repeated until the coordinate dependence of $\delta \Phi_{1}(y)$ is obtained. The $\delta \Phi_{1}(y)$ and $\delta \Phi_{2}(y)$ dependences are presented in Fig. 5. Fitting the experimental dependences $\delta \Phi_{1}(y)$ and $\delta \Phi_{2}(y)$ by a linear function $\delta \Phi=A y+B$, one can calculate the effective photoelastic coefficients:

$$
C_{1}^{e f f}=\frac{\lambda h^{3}}{12 \pi P a} A_{1}, \quad C_{2}^{e f f}=\frac{\lambda h^{3}}{12 \pi P a} A_{2} .
$$

Then the photoelastic coefficients $K_{\|}$and $K_{\perp}$ can be derived as 


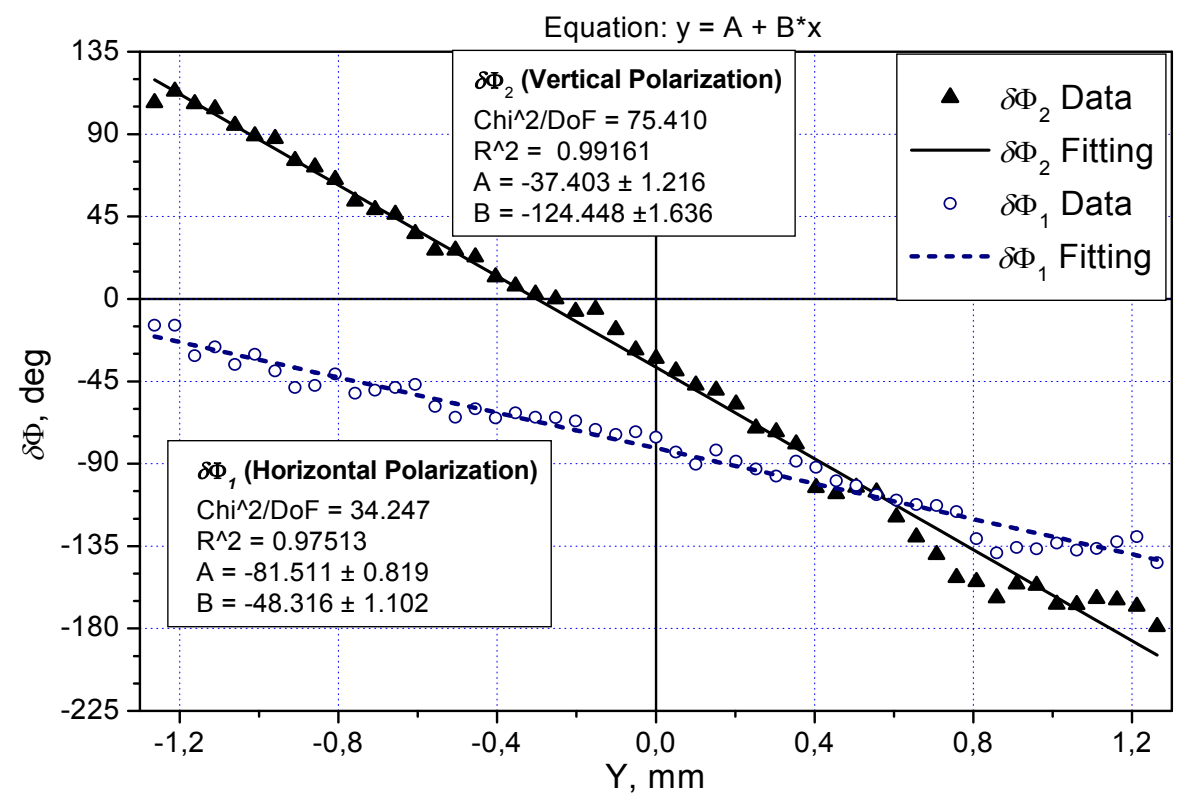

Fig. 5. Coordinate dependence of phase difference increments $\delta \Phi_{1}(y)$ and $\delta \Phi_{2}(y)$ fitted by linear functions. Fitting results and statistics are summarised in the legends.

$$
K_{\|}=C_{1}^{e f f}+(n-1) \frac{v}{E}, \quad K_{\perp}=C_{2}^{e f f}+(n-1) \frac{v}{E} .
$$

The stress optical coefficient $K=K_{\|}-K_{\perp}$ is a standard parameter tabulated for optical glasses. It is easily accessible from open manufacturers' data sheets and so its value can be used for checking validity of our technique. We have calculated the photoelastic coefficients $K_{\|}$and $K_{\perp}$, along with the stress optical coefficient $K$, for the two configurations of our interferometer. The first configuration has been adjusted for approximately five interference fringes within the fieldof-view, whereas the second one for approximately eight fringes. The values obtained for the coefficients $K_{\|}, K_{\perp}$ and $K$ (in the units of Brewster; $1 \mathrm{~B}=10^{-12} \mathrm{~Pa}^{-1}$ ) are presented in Table 1, to be compared with the reference data. As seen from Table 1, the reference data falls inside the confidence intervals for all the coefficients measured experimentally. In other words, our experimental results agree well enough with the available manufacturers' data. This indicates high accuracy of the method suggested in the present work. It is important that the relative error for the stress optical coefficient $K$ does not exceed $3 \%$, thus confirming high precision of both the experimental setup and the calculation procedures developed here.

Table 1. Photoelastic coefficients of standard optical glass BK7 calculated basing on our experiments, and the corresponding literature data.

\begin{tabular}{|c|c|c|c|}
\hline Coefficient & Configuration 1 & Configuration 2 & $\begin{array}{c}\text { Reference data } \\
\text { (for } \lambda=589 \mathrm{~nm})\end{array}$ \\
\hline$K_{\|}, \mathrm{B}$ & $-0.490 \pm 0.045$ & $-0.548 \pm 0.050$ & -0.5 (Ref. [24]) \\
\hline$K_{\perp}, \mathrm{B}$ & $-3.302 \pm 0.063$ & $-3.269 \pm 0.050$ & -3.3 (Ref. [24]) \\
\hline$K, \mathrm{~B}$ & $2.811 \pm 0.077$ & $2.722 \pm 0.071$ & 2.77 (Ref. [22]) \\
\hline
\end{tabular}


Finally, the piezooptic coefficients can be calculated using the measured values of the photoelastic coefficients $K_{\|}$and $K_{\perp}$ :

$$
\pi_{11}=-\frac{2 K_{\|}}{n^{3}}, \quad \pi_{12}=-\frac{2 K_{\perp}}{n^{3}} .
$$

We obtain the following piezooptic coefficients: $\pi_{11}=0.282 \pm 0.026 \mathrm{~B}$ and $\pi_{12}=1.899 \pm 0.036 \mathrm{~B}$ for the first configuration of our interferometer, and $\pi_{11}=0.315 \pm 0.029 \mathrm{~B}$ and $\pi_{12}=1.880 \pm 0.029 \mathrm{~B}$ for the second one. The average values are $\left\langle\pi_{11}\right\rangle=0.299 \mathrm{~B}$ and $\left\langle\pi_{12}\right\rangle=1.890 \mathrm{~B}$. The absolute error associated with repeatability reached for the piezooptic coefficients does not exceed $\pm 0.036 \mathrm{~B}$, thus corresponding to the best accuracy known from the literature $[20,25]$.

Unfortunately, the handbook [24] on optical materials does not contain the piezooptic coefficients for the optical glass BK7, providing instead its elastooptic coefficients $\left(p_{11}=0.12\right.$ and $p_{12}=0.22$ ). These coefficients are related to the piezooptic coefficients through the elastic modules $E$ and $v$ :

$$
p_{11}=\frac{E\left[(1-v) \pi_{11}+2 v \pi_{12}\right]}{(1+v)(1-2 v)}, \quad p_{12}=\frac{E\left(\pi_{12}+v \pi_{11}\right)}{(1+v)(1-2 v)} .
$$

Issuing from our experimental data, we find $p_{11}=0.118 \pm 0.004$ and $p_{12}=0.226 \pm 0.005$. These values also agree perfectly with the manufacturers' data, with the error levels being satisfactory from the standpoint of demands for the reference literature. Moreover, simple comparison testifies that the precision of our method is not worse than that of the Dixon-Cohen method, which is known to provide the best accuracy for the elastooptic coefficients [26, 27].

Besides, a significant advantage of our method is that it allows determining unambiguously the sign of both the piezooptic and photoelastic coefficients, unlike the canonical technique developed by Dixon and Cohen. Unambiguous determination of the signs of these coefficients is facilitated by the known signs of stresses and a proper calibration of interferometer. Though we have tested our instrumentation on an optical glass only, it seems to be a promising tool for studying piezooptic coefficients of crystalline materials.

\section{Conclusion}

In this work we have developed a new method for measuring the piezooptic coefficients, which combines digital imaging interferometry with the known four-point sample bending technique. The design of our interferometer, the measuring procedures, and the experimental data processing are described in detail. Exploiting one of the most popular optical glasses, BK7, as an example, we have successfully verified the capabilities of the method. High accuracy for measuring photoelasticity (namely, the piezooptic and elastooptic parameters) and low experimental errors of determination of the corresponding coefficients confirm good performance of instrumentation built by us. The absolute error determined by experimental uncertainty for the piezooptic coefficients does not exceed $\pm 0.036 \mathrm{~B}$. This is among the best figures known from the literature. At the same time, the error for the elastooptic coefficients does not exceed \pm 0.005 . An additional advantage of our method is unambiguous determination of signs of both the piezooptic and photoelastic coefficients.

\section{Acknowledgement}

One of the authors namely Oleg Krupych thanks Dr. I. Martynyuk-Lototska and Mr. T. Dudok for useful discussions. 


\section{References}

1. Brewster D, 1816. On the communication of the structure of doubly-refracting crystals to glass, murite of soda, flour spar, and other substances by mechanical compression and dilation. Phil. Trans.: 156-178.

2. Grakh I I and Mozhanskaya A F, 1971. A type of mechanically anisotropic, optically sensitive material. Mekhanika Polimerov. 5: 835-839.

3. Weber Y-J, 1995. Determination of internal strain by optical measurements. Phys. Rev. B 51: 12209-12215.

4. Narasimhamurty T S, Photoelastic and electrooptic properties of crystals. New York: Plenum Press (1981).

5. Slezinger I I, Alievskaya A N and Mironov Yu V, 1985. Piezooptic devices. Izmeritelnaya Tekhnika. 12: 17-19.

6. Billardon M and Badoz J, 1966. Birefringence modulator. C. R. Acad. Sci. Ser. B. 262: 16721675.

7. Kemp J C, 1969. Piezo-optical birefringence modulators: new use for a long-known effect. J. Opt. Soc. Amer. 59: 950-954.

8. Auld B A, Acoustic fields and waves in solids. Malabar, FL: Krieger (1990).

9. Balakshii V I, Parygin VN and Chirkov L E, Physical fundamentals of acoustooptics. Moscow: Radio i Sviaz' (1985).

10. Xu J and Stroud R, Acousto-optic devices: principles, design, and applications. New York: Wiley (1992).

11. Shaskolskaya M P, Acoustic crystals. Moscow: Nauka (1982).

12. http://www.schott.com/advanced_optics/us/abbe_datasheets/schott_datasheet_all_us.pdf, http://www.us.schott.com/advanced_optics/english/download/schott_tie-27_stress_in_optical _glass_july_2004_us.pdf

13. Pockels F, Lehrbuch der Kristallooptik. Leipzig: Teubner Berlin (1906).

14. Ajmera P K, Huner B, Dutta A K and Hartley C S, 1988. Simulation and observation of infrared piezobirefringent images in diametrically compressed semiconductor disks. Appl. Opt. 27: 752-757.

15. Andrushchak A S, Bobitski Ya V, Kaidan M V, Mytsyk B G, Kityk A V, Schranz W, 2005. Two-fold interferometric measurements of piezo-optic constants: application to $\beta-\mathrm{BaB}_{2} \mathrm{O}_{4}$ crystals. Opt. Laser Technol. 37: 319-328.

16. Mys O, Adamiv V, Martynyuk-Lototska I, Burak Ya and Vlokh R, 2007. Piezooptic and acoustic properties of $\mathrm{KLiB}_{4} \mathrm{O}_{7}$ crystals. Ukr. J. Phys. Opt. 8: 138-142.

17. Vasylkiv Yu, Kvasnyuk O, Krupych O, Mys O, Maksymuk O and Vlokh R, 2009. Reconstruction of 3D stress fields basing on piezooptic experiment. Ukr. J. Phys. Opt. 10: 22-37.

18. Frocht M M, Photoelasticity. London: J. Wiley and Sons (1965).

19. Vasylkiv Yu, Savaryn V, Smaga I, Skab I and Vokh R, 2010. Determination of piezooptic coefficient $\pi_{14}$ of $\mathrm{LiNbO}_{3}$ crystals under torsion loading. Ukr. J. Phys. Opt. 11: 156-164.

20. Skab I, Smaga I, Savaryn V, Vasylkiv Yu and Vlokh R, 2011. Torsion method for measuring piezooptic coefficients. Cryst. Res. \& Technol. 46: 23-36.

21. Timoshenko S P, Strength of materials. Moscow: Izdatelstvo NTL (1965).

22. http://edit.schott.com/advanced_optics/english/abbe_datasheets/schott_datasheet_n-bk7.pdf 
23. Mytsyk B H, 2003. Methods for the studies of the piezo-optical effect in crystals and the analysis of experimental data. Part I. Methodology for the studies of piezo-optical effect. Ukr. J. Phys. Opt. 4: 1-26.

24. Weber M J, Handbook of optical materials. Boca Raton, FL: CRC Press (2003).

25. Vasylkiv Yu, Savaryn V, Smaga I, Skab I and Vlokh R, 2011. On determination of sign of the piezo-optic coefficients using torsion method. Appl. Opt. 50: 2512-2518.

26. Dixon R W and Cohen MG, 1966. A new technique for measuring magnitudes of photoelastic tensor and its application to lithium niobate. Appl. Phys. Lett. 8: 205-207.

27. Dixon R W, 1967. Photoelastic properties of selected materials and their relevance for applications to acoustic light modulators and scanners. J. Appl. Phys. 38: 5149-5152.

Krupych O., Savaryn V., Skab I. and Vlokh R., 2011. Interferometric measurements of piezooptic coefficients by means of four-point bending method. Ukr.J.Phys.Opt. 12: 150-159.

Анотація. В даній роботі запропоновано новий метод вимірювання n'єзооптичних коефіцієнтів, який $\epsilon$ комбінацією цифрової лазерної інтерферометрії зображення $i$ відомого методу чотирьох-точкового згину. Детально описані конструкиія інтерферометра, прочедура вимірювання і обробка результатів. Можливості даного методу випробувані на прикладі, широко вживаного оптичного скла ВК7. Висока точність разом з можливістю визначення знаку, як n'єзооптичних $\left(\pi_{q m}\right)$ так $i$ фотопружних $\left(p_{q m}\right)$ коефіиієнтів дозволяє стверджувати, щзо запропонований метод є найточнішим і надійним при визначенні коефіцієнтів $\pi_{q m}$ i $p_{q m}$. 